\title{
Effect of Monocular Intravitreal Anti-Vascular Endothelial Growth Factor Injection on the Fellow Eye
}

\author{
Samah R. Abd El-Moniem*1, Ahmed I. Abou El-Enin ${ }^{2}$, Afaf H. Rashwan ${ }^{1}$
}

${ }^{1}$ Department of Ophthalmology, Faculty of Medicine for Girls, Al-Azhar University, ${ }^{2}$ Department of Ophthalmology, International Medical Center, Armed Forces, Cairo, Egypt. *Corresponding Authors: Samah Reda Abd El-Moniem; Tel.: (+20) 1009686203;

Email: samahsemsema92@gmail.com

\begin{abstract}
Background: The contralateral effect of intravitreal Anti-Vascular Endothelial Growth Factor (anti- VEGF) on the fellow eye is still controversial.

Objectives: The aim of the current work was to evaluate the contralateral effect of anti-VEGF injection on the fellow eye in patients with bilateral diabetic macular edema (DME).

Patients and Methods: This prospective interventional study included a total of 30 female patients with bilateral DME aged from 30 to 70 years, attending at Department of Ophthalmology, International Medical Center, Armed Forces. This study was conducted to assess the effect of unilateral intravitreal injection of anti-VEGF on the fellow eye. It was conducted between 2018 to 2019.

Results: The mean age \pm SD was $61.0 \pm 7.8$ years and duration of DM was $17.50 \pm 9.797$ years. All patients were obese with mean body mass index (BMI) $37.3 \pm 3.5$. 21 cases were hypertensive, 2 patients had albuminuria. Statistically significant decrease in central retinal thickness (CRT) in both eyes were found with mean \pm SD reduction $83.07 \pm 31.67 \mu \mathrm{m}$ in injected eye and $11.30 \pm 12.78 \mu \mathrm{m}$ in the untreated eye from baseline, no significant improvement in best corrected visual acuity (BCVA). There was no significance correlation between hypertension and improvement in CRT, while the patients with albuminuria showed worsening in CRT in untreated eye.

Conclusion: It could be concluded that ranibizumab can escape into the systemic circulation and reduce contralateral CRT and the systemic condition could affect the outcome of the treatment.
\end{abstract}

Keywords: DME, anti-VEGF, fellow eye.

\section{INTRODUCTION}

The incidence of diabetic retinopathy including diabetic macular edema (DME) which is the most common microvascular complication of DM, increased to alarming level and it considered the major causes of severe visual impairment in DM along with ${ }^{(\mathbf{1})}$.

Vitreous VEGF levels which is an important mediator in DM has been observed to be high in DME, using VEGF inhibitors (anti-VEGF) was believed to be beneficial in reversing the loss of vision caused by macular edema. Trials have handled the efficacy and safety of different types of anti-VEGF in the treatment of DME, including Pegaptanib, ranibizumab, bevacizumab, and aflibercept ${ }^{(2)}$.

However, the observation of fellow eye effects, along with the pharmacokinetic studies showing concentrations of the agents in the bloodstream correlating with reduction in circulating free VEGF levels, provide biologic possibility of potential systemic effects of these agents ${ }^{(3)}$.

So, it is suggested that anti-VEGF agents reach the untreated eye through the systemic circulation ${ }^{(4)}$, but the potential effect of unilateral injection of anti- VEGFs in the fellow eye is still doubtful in spite of some case reports of therapeutic effects after monocular injection of anti-VEGFs in the fellow eye ${ }^{(5)}$.

The aim of the current work was to evaluate the contralateral effect of anti-VEGF injection on the fellow eye in patients with bilateral diabetic macular edema (DME).

\section{PATIENTS AND METHODS}

This prospective interventional study included a total of 30 female patients with bilateral DME aged from 30 to 70 years, attending at Department of Ophthalmology, International Medical Center, Armed Forces. This study was conducted to assess the effect of unilateral intravitreal injection of anti-VEGF on the fellow eye. This study was conducted between 2018 to 2019.

\section{Ethical approval:}

Approval of the ethical committee was obtained.Written informed consent from all the subjects were obtained.

The inclusion criteria were diabetic patients with bilateral diabetic macular edema. The exclusion criteria were previous history of cataract surgeries in last six months, eyes with optic nerve pathology, previous macular edema treated by laser or anti-VEGF injections, previous history of subtenon or intraocular steroid injection, Presence of other retinal diseases and previous history of vitreo-retinal surgeries.

Full ophthalmic and medical history were taken. All patients were subjected to Full ophthalmological examination, visual acuity by snellen chart, pupils 
examination, intraocular pressure (IOP) measurement with Goldman applanation tonometer, Slit-lamp examination and Fundoscopic examination. Along with, color test by using Ishihara plats and Amselar grid test.

CRT measurement was performed bilaterally by spectral domain OCT (Spectralis, Heidelberg Engineering, Heidelberg, Germany), after appropriate pupillary dilatation using cyclopentolate $1 \%$ eye drops.

Systemic work up had done for patients before the injection including: BMI, fasting and postprandial serum glucose level that did not exceed $150 \mathrm{mg} / \mathrm{dl}$ for fasting and $200 \mathrm{mg} / \mathrm{dl}$ for postprandial, $\mathrm{HbA}_{1 \mathrm{C}}$, Control hypertension so the systolic blood pressure did not exceed $140 \mathrm{mmHg}$ and diastolic blood pressure 90 $\mathrm{mmHg}$, INR was done before the injection and had to be within normal range, anticoagulant/ antiplatelet agents was stopped before the injection for at least 3 days, liver and kidney functions and $\mathrm{CBC}$.

All patients were scheduled to receive 1 intravitreal injection of $0.5 \mathrm{mg}$ ranibizumab. Intravitreal injections were performed in the eye exhibiting more severe macular edema, which was determined by the central thickness and visual acuity.

All intravitreal injections were performed according to a standard protocol at the operation theater. 4 weeks after the injection same systemic laboratory investigations, BCVA and OCT were repeated.

\section{Statistical analysis}

Recorded data were analyzed using the statistical package for social sciences, version 20.0 (SPSS Inc., Chicago, Illinois, USA). Quantitative data were expressed as mean \pm standard deviation (SD). Qualitative data were expressed as frequency and percentage.

\section{The following tests were done:}

- Independent-samples t-test of significance was used when comparing between two means.

- Chi-square $\left(\mathrm{x}^{2}\right)$ test of significance was used in order to compare proportions between two qualitative parameters.

- The confidence interval was set to $95 \%$ and the margin of error accepted was set to $5 \%$. The pvalue was considered significant as the following:

- Probability (P-value)

- P-value $<0.05$ was considered significant.

- P-value <0.001 was considered as highly significant.

- P-value >0.05 was considered insignificant.

\section{RESULTS}

Thirty female patients suffering from DM were enrolled into this study with bilateral NPDR with macular edema received single injection in the worst eye. We chose patients with normal systemic lab investigations pre-injection ranges as demonstrated in table (1).

Table (1): Baseline demographics and ocular characteristics of patients pre and post injection:

\begin{tabular}{|c|c|c|}
\hline & Mean \pm SD & \\
\hline & Pre-injection & Post-injection \\
\hline Age (years) & $61.0 \pm 7.8$ & \\
\hline Duration of DM (years) & $17.50 \pm 9.797$ & \\
\hline Body mass index $\left(\mathrm{kg} / \mathrm{m}^{2}\right)$ & $37.3 \pm 3.5$ & \\
\hline HbA1c (\%) & $7.37 \pm 0.399$ & \\
\hline hemoglobin (g/dl) & $11.93 \pm 0.455$ & $11.90 \pm 0.430$ \\
\hline \multicolumn{3}{|l|}{ Kidney functions } \\
\hline Urea (mg/dl) & $22.57 \pm 7.001$ & $23.30 \pm 7.091$ \\
\hline Creatinine (mg/dl) & $0.82 \pm 0.137$ & $0.85 \pm 138$ \\
\hline \multicolumn{3}{|l|}{ Liver functions } \\
\hline SGOT (U/L) & $20.73 \pm 5.311$ & $21.60 \pm 5.150$ \\
\hline SGPT (U/L) & $25.80 \pm 6.266$ & $26.47 \pm 6.067$ \\
\hline INR & $1.01 \pm 0.19$ & $1.01 \pm 0.037$ \\
\hline $\begin{array}{l}\text { Fasting serum sugar } \\
(\mathbf{m g} / \mathbf{d l})\end{array}$ & $96.00 \pm 8.400$ & $123.67 \pm 22.350$ \\
\hline $\begin{array}{l}\text { Post prandial serum } \\
\text { sugar(mg/dl) }\end{array}$ & $123.67 \pm 22.350$ & $129.73 \pm 8.043$ \\
\hline IOP (mmHg) & RT $12.50 \pm 2$, LT $12.7 \pm 2.4$ & RT $13.2 \pm 1.7$, LT $13.5 \pm 2.1$ \\
\hline
\end{tabular}

We divided our patients into 3 groups as showed in table (2), 2 cases of group 3 were having positive albuminuria in urine analysis. 
Table (2): Distribution of cases according to the change in CRT of the fellow eye:

\begin{tabular}{llll}
\hline & \multicolumn{2}{l}{ Change in fellow eye's CRT } & Number of cases \\
\hline Group 1 & Improved & Decreased $>10 \mu \mathrm{m}$ & 17 \\
Group 2 & Stable & $\pm 10 \mu \mathrm{m}$ & 10 \\
Group 3 & Worsened & Increased $>10 \mu \mathrm{m}$ & 3 \\
\hline
\end{tabular}

Statistically significant changes were found after injection in both eyes the mean CRT of injected eye preinjection was $432.63 \pm 107.158 \mu \mathrm{m}$ and decreased significantly post injection to $349.57 \pm 99.910 \mu \mathrm{m}$ with mean reduction $83.07 \pm 31.67 \mu \mathrm{m}$, The mean CRT of fellow eye preinjection348.90 $\pm 97.431 \mu \mathrm{m}$ and became $336.93 \pm 97.060 \mu \mathrm{m}$ post injection with mean reduction in CRT $11.30 \pm 12.78 \mu \mathrm{m}$ as showed in figures (1) and tables $(2,3,4,5)$.
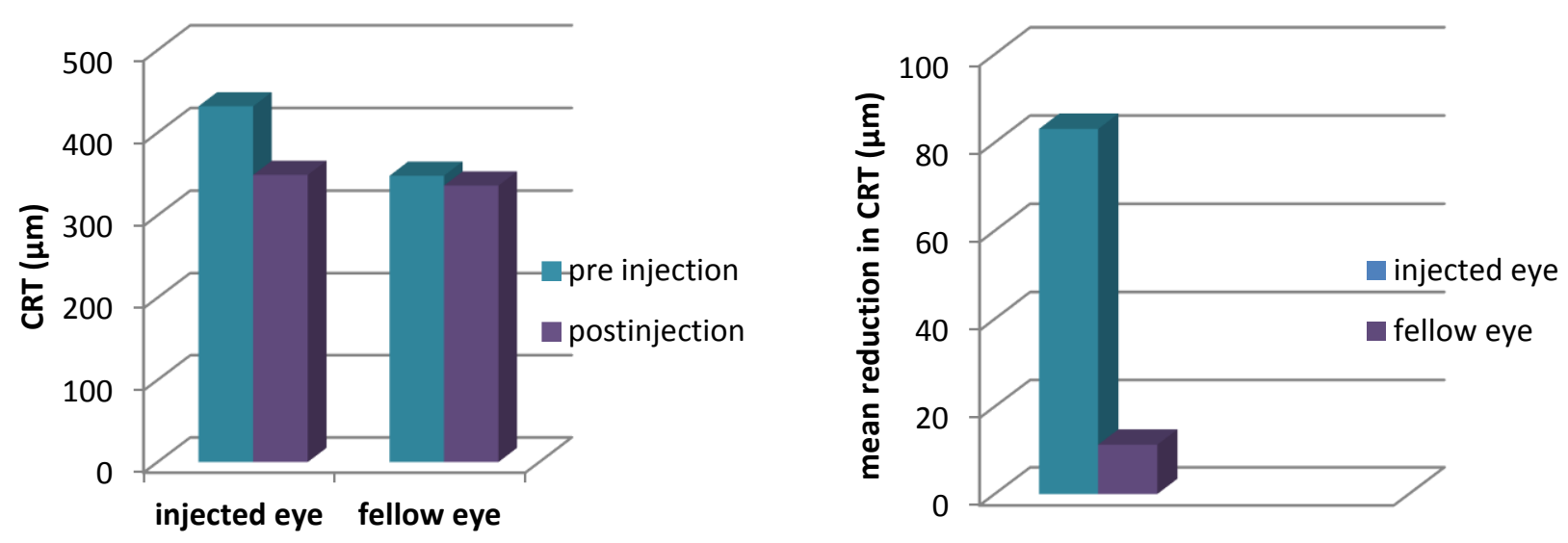

Figure (1): shows the improvement in CRT of both eyes post-injection (the chart on the left) and the correlation between the mean reduction of CRT of both eyes (the chart on the right).

Table (3): The mean improvement in CRT of injected eye and it's significance.

\begin{tabular}{llccc}
\hline & & Mean \pm SD & T & P value \\
\hline Injected eye & Pre-injection & $432.63 \pm 107.158 \mu \mathrm{m}$ & 29 & 0.0001 \\
& Post-injection & $349.57 \pm 99.910 \mu \mathrm{m}$ & & \\
\hline
\end{tabular}

Table (4): The mean improvement in CRT of fellow eye and it's significance.

\begin{tabular}{llccc}
\hline & & Mean \pm SD & T & P value \\
\hline Fellow eye & Pre-injection & $348.90 \pm 97.431 \mu \mathrm{m}$ & 29 & 0.0001 \\
& Post-injection & $336.93 \pm 97.060 \mu \mathrm{m}$ & & \\
\hline
\end{tabular}

Table (5): the mean reduction of CRT in both eyes:

\begin{tabular}{ccc}
\hline & Injected eye & Fellow eye \\
\hline Mean \pm SD & $83.07 \pm 31.67$ & $11.30 \pm 12.78$ \\
\hline
\end{tabular}


ejhm.journals.ekb.eg

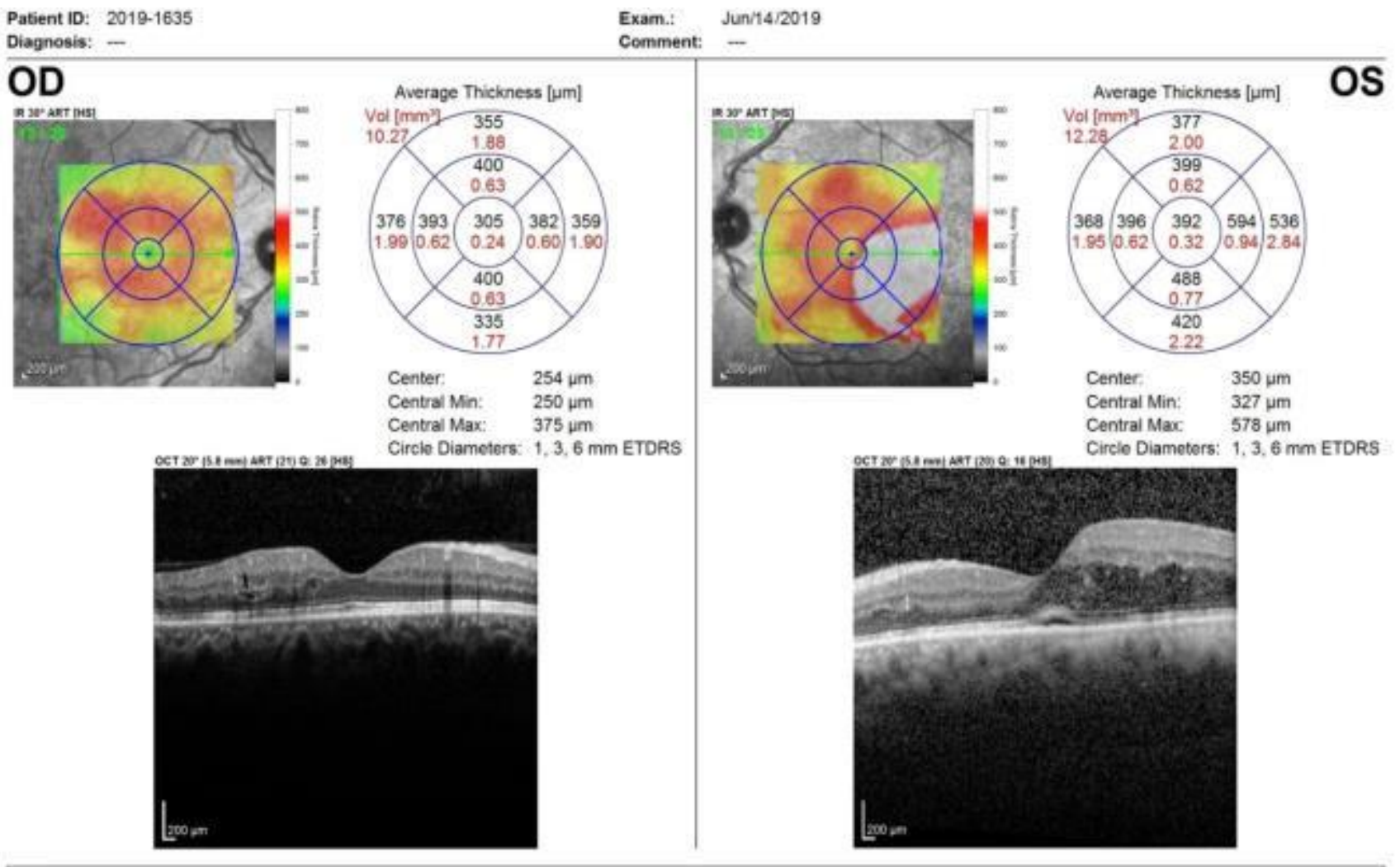

Figure (2): case no.1 (group 1) showing bilateral cystoid diabetic macular edema pre-injection, the BCVA was $6 / 9$ and $6 / 36$ by snellen chart for right and left eye respectively.

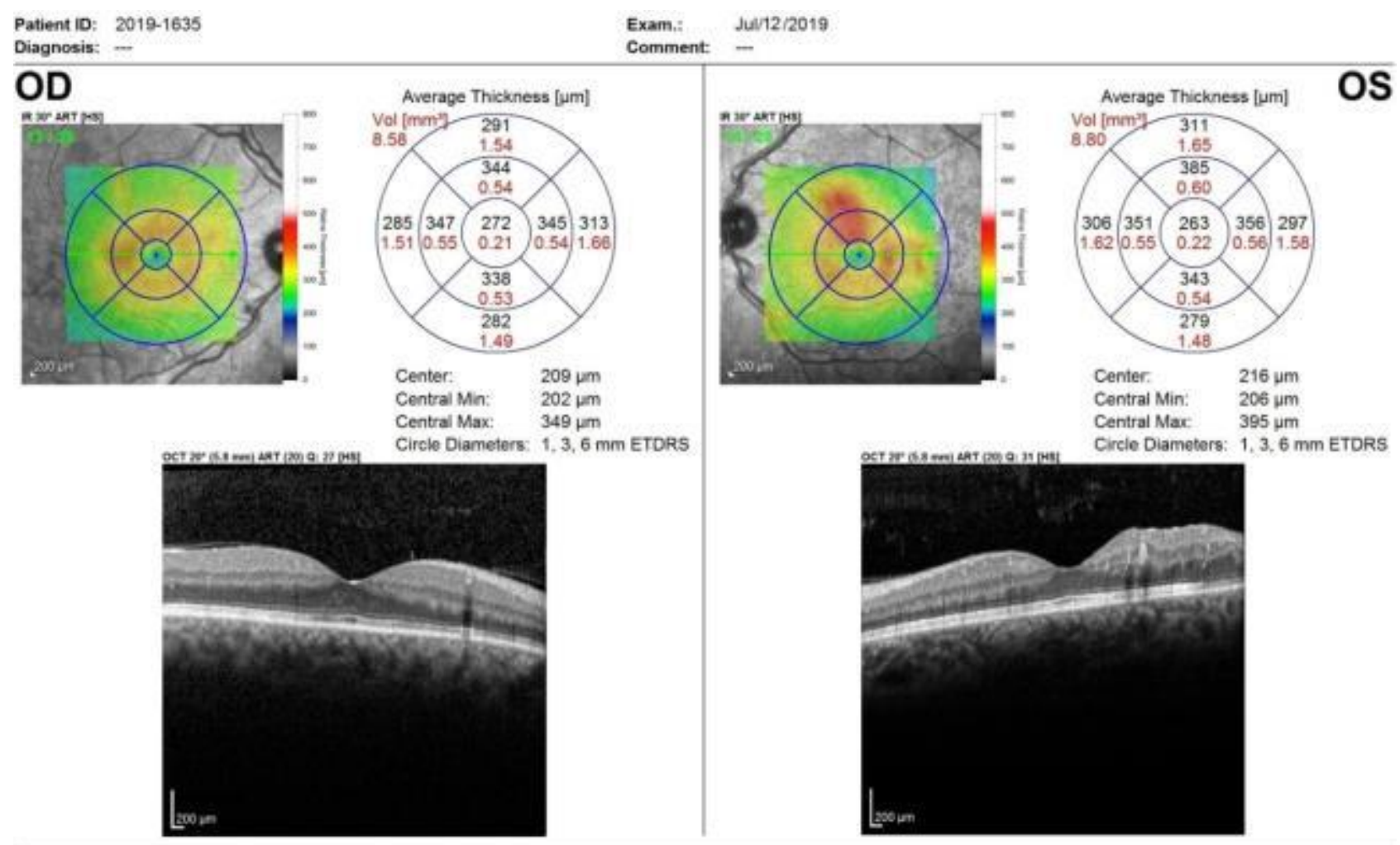

Figure (3): case no.1 (group 1) after injecting the left eye showing decrease CRT in both eyes and BCVA improved in left eye to $6 / 12$ while remained $6 / 9$ in right eye. 
ejhm.journals.ekb.eg



Figure (4): case no.10 (group 2) showing bilateral cystoid diabetic macular edema pre-injection, the BCVA was $6 / 9$ and $6 / 12$ by snellen chart for right and left eye respectively.

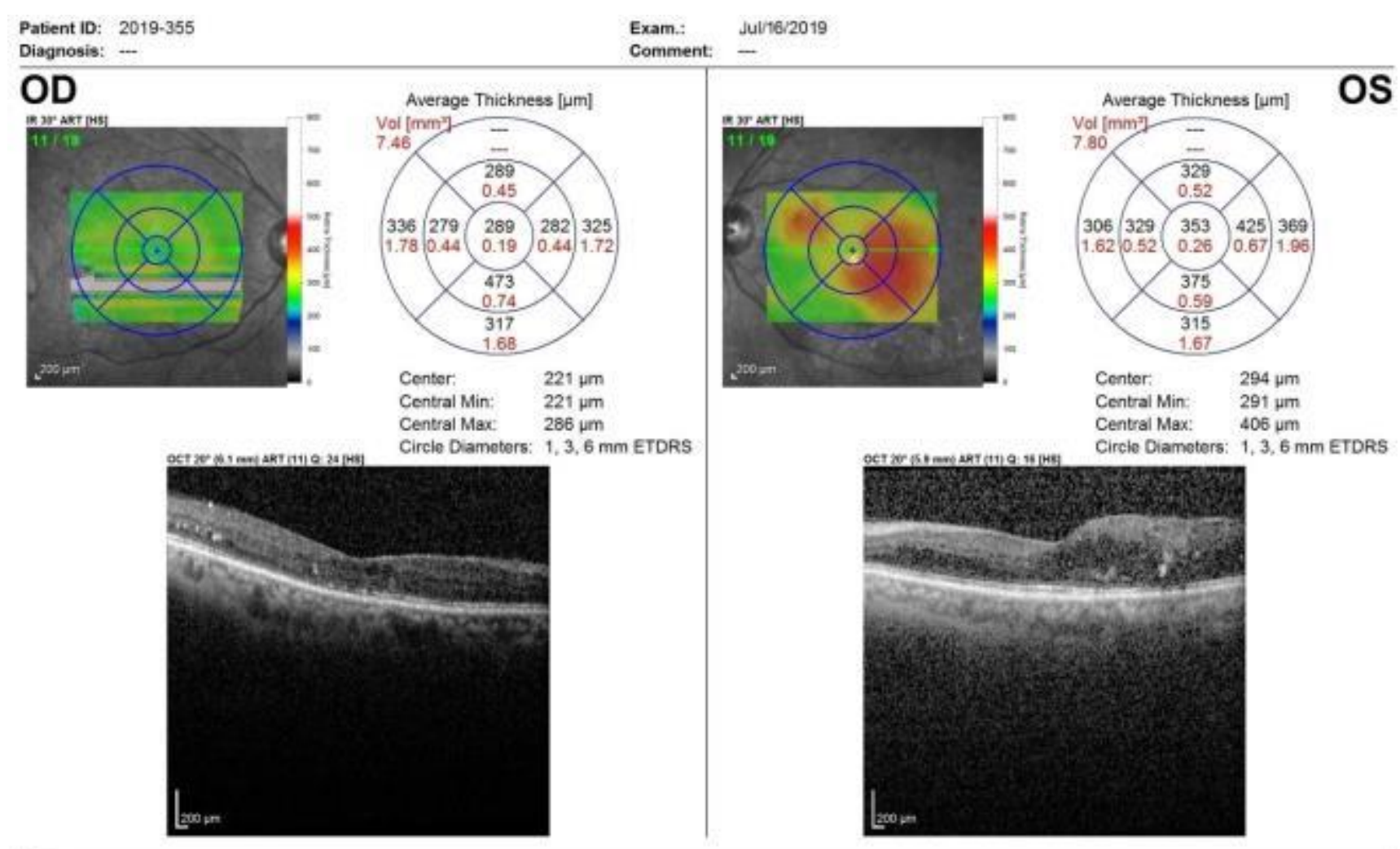

Figure (5): case no.10 (group 2) after injecting the left eye showing decrease CRT in the injected eye while the right eye (the fellow eye) showed increase in CRT but only $10 \mu \mathrm{m}$ and BCVA remained unchanged in both eyes. 


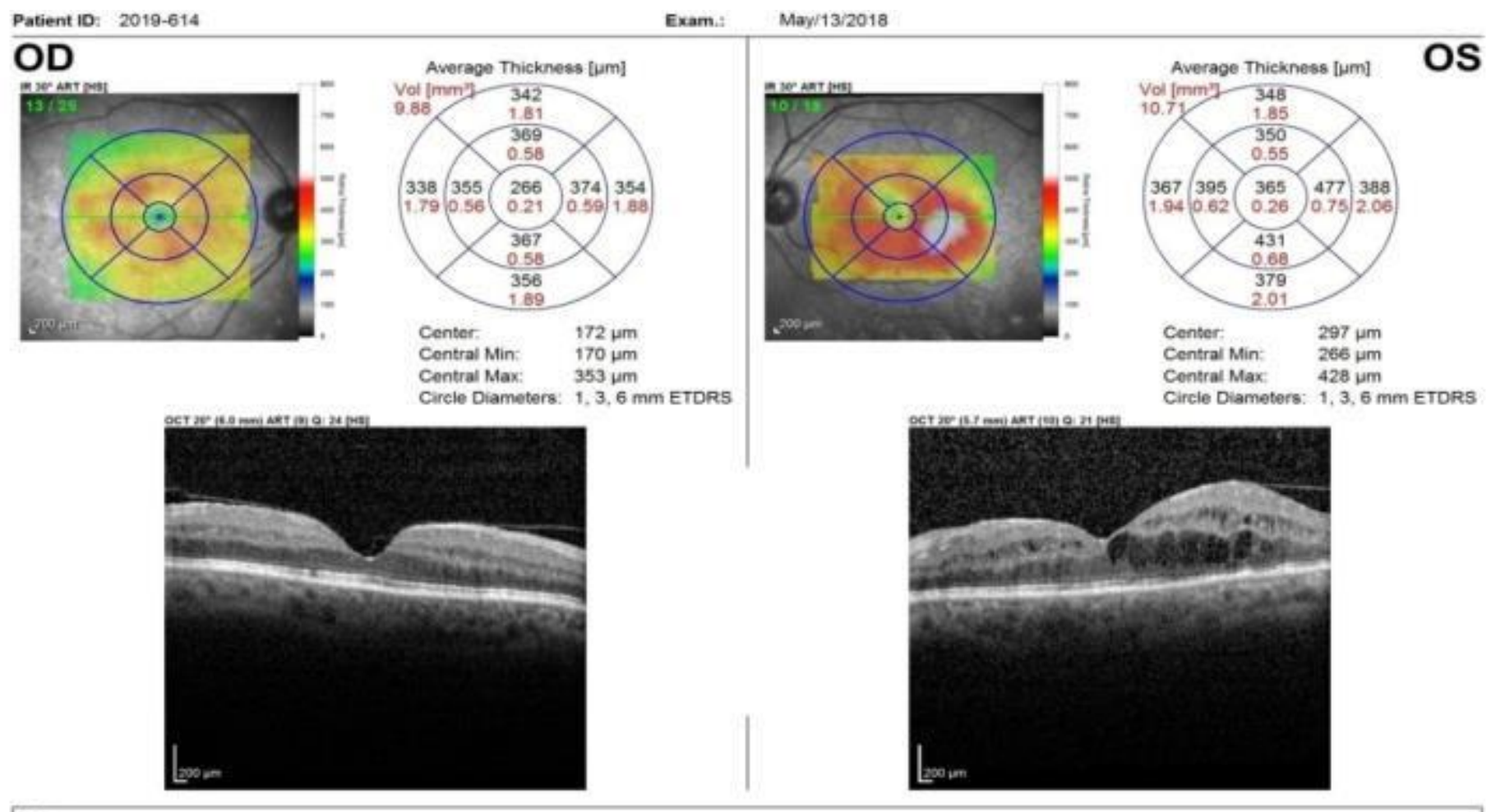

Figure (6): case no.19 (group 3) with positive albuminurea had right diffuse DME and left cystoid DME preinjection. The BCVA $6 / 9$ and 6/12 for the right and left eye respectively.

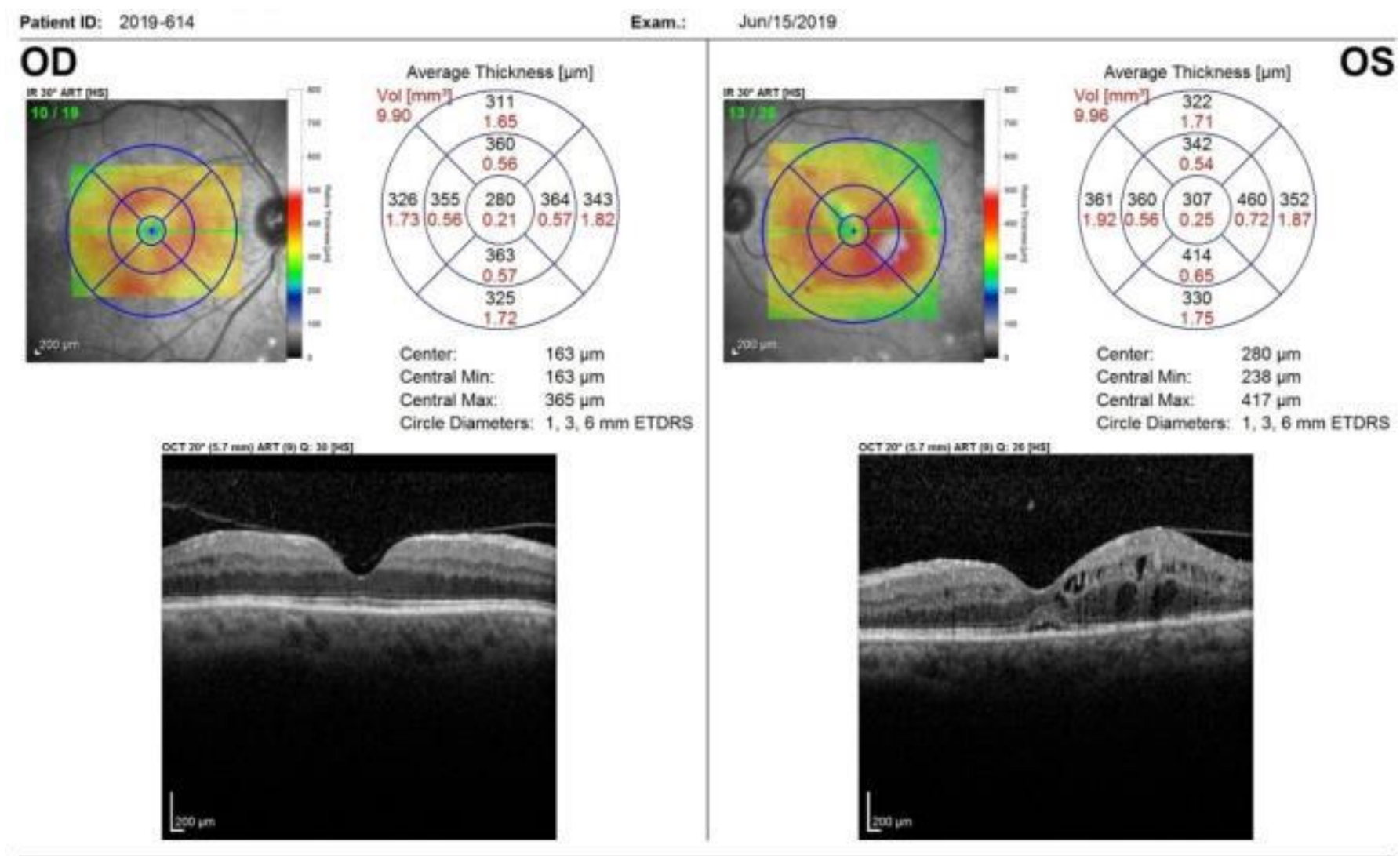

Figure (7): case no.19 (group 3) after injection of the left eye, showing improvement in CRT of left eye (injected eye) while the CRT of the right eye (untreated eye) increased. The BCVA of both eyes didn't Mean best corrected visual acuity (BCVA) by ((LogMAR) approximate snellen equivalent) significantly improved in injected eye but remain unchanged in the fellow eye as showed in table (6) and (7). 
Table (6): The mean BCVA in the injected eye pre and post injection and it's significance.

\begin{tabular}{lllll}
\hline & & Mean \pm SD & T & P value \\
\hline \multirow{2}{*}{ Injected eye } & Pre-injection & $0.51 \pm 0.215(6 / 18)$ & 29 & 0.0001 \\
& Post-injection & $0.33 \pm 0.180(6 / 12)$ & & \\
\hline
\end{tabular}

Table (7): The mean BCVA in the fellow eye pre and post injection and it's significance.

\begin{tabular}{cllll}
\hline & & Mean \pm SD & T & P value \\
\hline \multirow{2}{*}{ fellow eye } & Pre-injection & $0.34 \pm 0.229(6 / 12)$ & 29 & 0.476 \\
& Post-injection & $0.34 \pm 0.239(6 / 12)$ & & \\
\hline
\end{tabular}

On examination there were no significant abnormalities in anterior segment, all the patients had normal color vision by ishihara plates. Amselar grid was normal in Twenty-six cases while four cases were distorted.

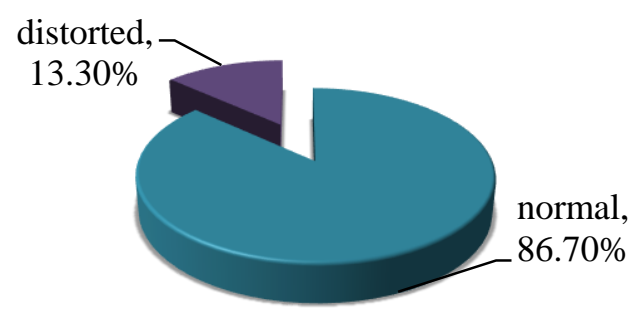

Figure (8): Amselar grid distribution among the patients.

$70 \%$ of cases were hypertensive ( 21 cases) and $30 \%$ normotensive ( 9 cases) and $93.3 \%$ of cases without albuminurea (28 cases) $6.7 \%$ with albuminurea ( 2 cases) as showed in. There is no statistically significance correlation between hypertension and the change of CRT in both eyes. As showed in tables (8), (9), (10).

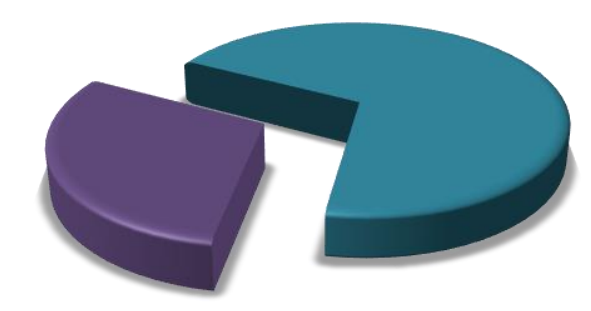

anormotensive $30 \%$ ahypertensive $70 \%$

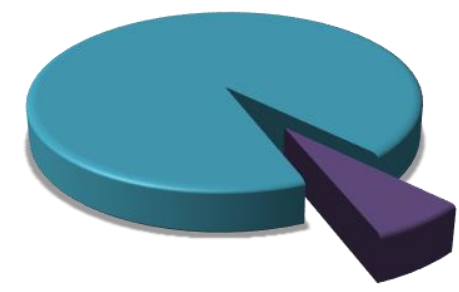

with albuminurea $93.33 \%$

without albuminurea $6.67 \%$

Figure (9): distribution of hypertension and albuminurea distribution in the study.

Table (8): Correlation between hypertension and reduction in CRT of Injected eyes and its significance:

\begin{tabular}{llll}
\hline & Mean \pm SD & T & P value \\
\hline Hypertensive & $88.14 \pm 34.26$ & 1.37 & 0.183 \\
normotensive & $71.22 \pm 22.16$ & & \\
\hline
\end{tabular}

Table (9): Correlation between hypertension and reduction in CRT of Fellow eyes and its significance:

\begin{tabular}{llll}
\hline & Mean \pm SD & T & P value \\
\hline Hypertensive & $11.76 \pm 14.8$ & 0.298 & 0.768 \\
normotensive & $10.22 \pm 6.3$ & & \\
\hline
\end{tabular}

Table (10): The correlation between albuminurea and the change in the fellow eye:

\begin{tabular}{llll}
\hline & -ve albuminurea & +ve albuminurea & Total \\
\hline Group 1 & 17 & 0 & 17 \\
Group 2 & 10 & 0 & 10 \\
Group 3 & 1 & 2 & 3 \\
\hline
\end{tabular}




\section{DISCUSSION}

Anti-VEGF antibodies nowadays considered the first-line treatment in DME. Reaching Anti-VEGF drugs administered through intravitreal route in human's eyes the systemic circulation is a fact which has been widely accepted.

This is supported by few studies regarding the pharmacokinetics and distribution of these agents after intravitreal injection that demonstrating decrease in VEGF levels in the blood after intravitreal injections as Sato et $\boldsymbol{a l} .{ }^{(6)}$ have found significant decrease in serum VEGF levels 1 week after injecting $0.5 \mathrm{mg}$ IVB. Carneiro et al. ${ }^{(7)}$ showed a $42 \%$ decrease in serum VEGF after a third IVB in patients with neovascular AMD. Zehetner et al. ${ }^{(8)}$ also found similar effects after IVB,

Zehetner et al. ${ }^{(9)}$ conducted another study on aflibercept which gave a similar effect on systemic VEGF level. Along with Hoerster et al. ${ }^{(10)}$ who reported that serum VEGF levels in infants with ROP reduced after 2weeks from injection of 0.2 mgofranibizumab.

Our study demonstrates the presence of statistically significant effect of a single $0.5 \mathrm{mg}$ ranibizumab injection on CRT of the contralateral but no significant improvement on BCVA. We selected patients with bilateral DME with no other ocular diseases and both eyes had didn't receive any treatment in the past. The worst eye diagnosed clinically through fundus examination and visual acuity and the CRT by OCT. They received a single injection and followed 4 weeks after by visual acuity and OCT.

\section{$>\quad$ Central macular thickness:}

According to CRT changes our results agreed with that of Sharma and Ong et al. ${ }^{(11)}$, as they reported a case showed a significant improvement in macular thickness of the non-injected eye of a patient receiving unilateral ranibizumab for diabetic macular edema. Rotsos et al. ${ }^{(\mathbf{1 2})}$, reported a case with bilateral DME who showed significant anatomical improvement and reduction in CRT was observed in the fellow eye after 2 intravitreal ranibizumab injections in one eye.

There are studies demonstrated that there is contralateral effect in DME but with others antiVEGF agents as Rahimy et al. ${ }^{(13)}$, reported a case with bilateral diffuse DME received single unilateral intravitreal aflibercept injection and showed bilateral response and improvement in CRT and Bakbak et al. (14) reported in patients with bilateral DME. A significant contralateral effect but after intravitreal bevacizumab injection in one eye

Other studies showed the contralateral effect of ranibizumab in other diseases as Wu and Sadda ${ }^{(15)}$ reported a case with branch retinal vein occlusion
(BRVO) in which ranibizumab appeared to have effects in the contralateral untreated eye. Another study conducted by Acharya et al. ${ }^{(16)}$ demonstrated a beneficial effect of ranibizumab in both eyes of patients ( 2 out of 3 patients) who were treated with unilateral ranibizumab injection but for uveitisrelated CME and Pescosolido et al. ${ }^{\left({ }^{(17)}\right.}$ who reported a case with bilateral subfoveal choroidal neovascularization, the case was injected with ranibizumab showed remission of the CNV of both eyes but after the second injection.

Our results not agreed with that of Bakbak et al. (14), who found statistically significant decrease in CRT in untreated eyes after treatment with bevacizumab, but not ranibizumab. Recently Malbin et al. ${ }^{(18)}$ conducted a comparative study to see the contralateral effect of the three anti-VEGF agents, bevacizumab, ranibizumab and aflibercept, in patients with bilateral DME and found only aflibercept that reduced the CRT of the fellow eye significantly.

Also, there are studies demonstrated that ranibizumab had no effect on the fellow eye in other retinal diseases as Gamulescu and Helbig ${ }^{\left({ }^{(9)}\right.}$, who observed no therapeutic effect of ranibizumab in the untreated fellow in patients with ARMD.

While in the same year to see the effect of antiVEGF agents on the fellow eye in patients with DME Velez-Montoya et al. $^{(20)}$ designed a study and found no significant change in CRT of the untreated eye 4 weeks after bevacizumab injection in one eye in patients with bilateral DME.

$>$ Visual acuity:

Our results of the BCVA agreed with Bakbak et al. ${ }^{(14)}$ as he evaluated a crossover effect of ranibizumab in patients with bilateral DME. He have found no statistically significant change on BCVA in the contralateral eye, in patients with DME. Malbin et al. (18) supported our results in his study that mentioned before and found the change between the initial visual acuity and 1-month after the injection in the 3 fellow-eye groups was not statistically significant.

And about the concept of the effect of antiVEGF on the fellow eye we are in line with Hanhart et al. ${ }^{(21)}$ as he evaluated the effect of bevacizumab in patients with bilateral DME and found there was no significant improvement of the VA in the noninjected eye.

On the other hand, our results did not agree with Rotsos et al. ${ }^{(12)}$, who reported a significant visual improvement in the fellow eye after 2 ranibizumab injections in their case report.

But according to other retinal diseases Rouvas et al. ${ }^{(22)}$ conducted a retrospective study in patients with ARMD and demonstrated visual improvement in contralateral eye after intravitreal 
ranibizumab injection. And Pescosolido et al. (17) also reported a statistically significant improvement in BCVA untreated eye of the patient with $\mathrm{CNV}$ after the second ranibizumab injection.

It has been observed that the responses to antiVEGF treatments are very variable among diabetic patients and unpredictable. Kim et al. ${ }^{(23)}$ have found that the response to the treatment strategies may be affected by DME patterns determined by OCT.

So, it can be suggested that a good understanding of if the systemic risk factors may affect the morphology of the macula in DME so that would help predict treatment outcome.

Acan et al. ${ }^{(24)}$, found that Micro-albuminurea or macro-albuminurea may be more frequent and $\mathrm{HbA1c}$ level may be higher in patients with Diffuse macular edema.

In our study we noticed that patients with albuminurea their CRT of the fellow eye increased post-injection and the injected eye's CRT improvement was less than patients without albuminurea. So, it can be suggested that the systemic condition of diabetic patients may affects their response to injection as the albuminurea is a major risk factor in development of DME.

But we didn't find any significance relation between hypertension and the improvement of the fellow eye. This may agree with Chew ${ }^{(25)}$, who discussed the results of the ACCORD Eye Study (Action to Control Cardiovascular Risk in Diabetes), a randomized controlled clinical trial that examined the benefits of intense blood glucose control, blood pressure, and serum lipid levels on retinopathy in type 2 diabetes patients.

And her clinical recommendations were that intensive glycemic control are important in the treatment of DR but Intensive blood pressure control (that the systolic to be below $140 \mathrm{~mm} \mathrm{Hg}$ ) may not be necessary because of the weak evidence of benefit from blood pressure control in the progression of DR (2).

The mechanism of effects of injection antiVEGF agents on the contralateral eye is controversial till now. The molecule has been shown to pass into the eyes from the systemic circulation after systemic use of bevacizumab in patient with ARMD according to Michels et al. ${ }^{(26)}$.

To explain the effect in the untreated eye, Pescosolido et al. ${ }^{(17)}$ hypothesized a neuronal or systemic diffusion of ranibizumab that is able to induce the same effects in the contralateral eye as in the treated eye. Ranibizumab is smaller in molecular weight in comparison with bevacizumab, so it is possible that it may reach the contralateral eye more easily through the systemic circulation.
However, animal studies have shown that while both bevacizumab and ranibizumab were found in small amounts in the serum of rabbits after intravitrealadministration, only bevacizumab reached and was found in the aqueous of the fellow eye Acharya et al. ${ }^{(16)}$.

We are not sure about why we found a fellow eye effect in our patients treated with unilateral ranibizumab. Our theory is that the control of systemic risk factors such as $\mathrm{HbA1c}$ and hypertension along with kidney functions is the main reason this theory supported by our results about the patients with albuminuria whom their CRT increased after the injection.

If we took serum assays and vitreous or aqueous samples before and after intravitreal injection, we would have a better idea about the pharmacokinetics of ranibizumab. However, at the time of designing this study, we believed that these were invasive procedures for our study, which was aimed at identifying changes in BCVA and CRT

And about the lack of BCVA effect we believed that because we only administered single injection as it has been suggested that the altered blood-retina barrier resulted from diabetes may require multiple doses of anti-VEGF injections before maximal clinical response can be seen in DME

However, our results support the hypothesis that ranibizumab treatment in one eye may have a contralateral effect and this will have a lot of benefits to the patients and decrease the cost.

The short follow-up time, small sample size, and the absence of a control group of untreated patients are the major limitations in our study. Along with the shortage in similar literatures that focus on the potential therapeutic effect of intravitreal ranibizumab in the fellow eye in patients with bilateral DME as most of literatures focused mainly on exudative AMD and the bevacizumab agent.

\section{CONCLUSION}

It could be concluded that ranibizumab can escape into the systemic circulation and reduce contralateral CRT and the systemic condition could affect the outcome of the treatment.

\section{REFERENCES}

1. Tarr JM, iKaul K, Chopra M et al. (2013): Pathophysiology of Diabetic Retinopathy. ISRN Ophthalmol., 2013: 1-13.

2. Stefanini FR, Badaró E, Falabella $P$ et al. (2014): Anti-VEGF for the Management of Diabetic Macular Edema. J Immunol Res., 2014:1-8.

3. Roman K (2015): Examining the Systemic Safety of Anti-VEGF Agents. Retina today, 15: 81-4.

4. Michalska-Malecka K, Kabiesz A, Kimsa MW et al. (2016): Effect of intravitreal ranibizumab on the 
untreated eye and systemic gene expression profile in age-related macular degeneration. Clin Interv Aging, 11: 357-65.

5. Torabi H (2017): Improvement of Diabetic Macular Edema in the Fellow Eye After Monocular Intravitreal Bevacizumab Injection. Hospital Practices and Research HPR., 2 (2): 54-55.

6. Sato T, Wada K, Arahori H et al. (2012): Serum concentrations of bevacizumab (avastin) and vascular endothelial growth factor in infants with retinopathy of prematurity. Am J Ophthalmol., 153(2):327-33.

7. Carneiro AM, Costa R, Falcão MS et al. (2012): Vascular endothelial growth factor plasma levels before and after treatment of neovascular age-related macular degeneration with bevacizumab or ranibizumab. Acta Ophthalmol., 90(1):25-30

8. Zehetner C, Kirchmair R, Huber S et al. (2013): Plasma levels of vascular endothelial growth factor before and after intravitreal injection of bevacizumab, ranibizumab and pegaptanib in patients with age-related macular degeneration, and in patients with diabetic macular oedema. Br J Ophthalmol., 97(4):454-9.

9. Zehetner C, Kralinger M T, Modi YS (2015): Systemic levels of vascular endothelial growth factor before and after intravitreal injection of aflibercept or ranibizumab in patients with age-related macular degeneration: a randomised, prospective trial. Acta Ophthalmol., 93 (2):154-9.

10. Hoerster R, Muether P, Dahlke C et al. (2013): Serum concentrations of vascular endothelial growth factor in an infant treated with ranibizumab for retinopathy of prematurity. Acta Ophthalmol., 91(1):74-5.

11. Sharma NS, Ong JM, Ooi JL (2015): Re: 'Fellow eye effect of unilateral intravitreal bevacizumab injection in eyes with diabetic macular edema'. Eye (Lond), 29(2): 291-2.

12. Rotsos T, Symeonidis C, Triantafillopoulou $\mathrm{L}$ et al. (2014): Significant reduction of diabetic macular edema following intravitreal 1 ranibizumab injection in the fellow eye. Int Ophthalmol., 34(6): 1271-4.

13. Rahimy E, Nyong'o O, Leng T (2017): Significant Bilateral Response in Diabetic Macular Edema After Single Unilateral Intravitreal Aflibercept Injection. Ophthalmic Surg Lasers Imaging Retina, 48(2):167-9.

14. Bakbak B, Ozturk BT, Gonul S et al. (2016): The effect of intravitreal bevacizumab and ranibizumab on macular edema of the contralateral eye: A comparative study of two anti-VEGFs. Oman J Ophthalmol., 9(1):448.

15. Wu $Z$ and Sadda SR (2008): Effects on the contralateral eye after intravitreal bevacizumab and ranibizumab injections: a case report. Ann Acad Med Singapore, 37(7): 591-3.

16. Acharya NR, Sittivarakul W, Qian Y et al. (2011): Bilateral effect of unilateral ranibizumab in patients with uveitis-related macular edema. Retina, 31(9):18716

17. Pescosolido N, Fazio S, Rusciano D (2014): Therapeutic Improvement in the Contralateral Eye after Ranibizumab Intravitreal Treatment in a Patient Affected by Bilateral Subfoveal Choroidal Neovascularization: a case report. JSM Biotechnol Bioeng., 2(2): 1038.

18. Malbin B, Pate MB, He Y et al. (2019): Comparative Eye Effect of Unilateral Intravitreal Bevacizumab, Ranibizumab, and Aflibercept for Diabetic Macular Edema. Journal of Vitreo Retinal Diseases, 3(2) 86-9.

19. Gamulescu MA and Helbig H (2010): Lack of Therapeutic Effect of Ranibizumab in Fellow Eyes After Intravitreal Administration. J Ocul Pharmacol Ther., 26 (2): 213-6.

20. Velez-Montoya R, Fromow-Guerra J, Burgos O et al. (2009): The effect of unilateral intravitreal bevacizumab (avastin), in the treatment of diffuse bilateral diabetic macular edema: a pilot study. Retina, 29(1):20-6.

21. Hanhart J, Tiosano L, Averbukh E et al. (2014): Fellow eye effect of unilateral intravitreal bevacizumab injection in eyes with diabetic macular edema. Eye (Lond), 28(6): 646-53.

22. Rouvas A, Liarakos VS, Theodossiadis $P$ et al. (2009): The Effect of Intravitreal Ranibizumab on the Fellow Untreated Eye with Subfoveal Scarring due to Exudative Age-Related Macular Degeneration. Ophthalmologica, 223(6):383-9.

23. Kim M, Lee P, Kim Y et al. (2011): Effect of Intravitreal Bevacizumab Based on Optical Coherence Tomography Patterns of Diabetic Macular Edema. Ophthalmologica, 226(3):138-44.

24. Acan D, Karahan E, Kocak N et al. (2018): Evaluation of systemic risk factors in different optical coherence tomographic patterns of diabetic macular edema. Int $\mathbf{J}$ Ophthalmol., 11(7): 1204-9.

25. Chew E (2010): The ACCORD Study group, ACCORD Eye Study Group. Effects of medical therapies on retinopathy progression in type 2 diabetes. $\mathrm{N}$ Engl $\mathrm{J}$ Med., 363(3): 233-44.

26. Michels S, Rosenfeld PJ, Puliafito CA et al. (2005): Systemic bevacizumab (Avastin) therapy for neovascular age-related macular degeneration twelveweek results of an uncontrolled open-label clinical study. Ophthalmology, 112(6):1035-47. 